\title{
Monocular depth ordering with occlusion edges extraction and local depth inference
}

\author{
SONG Guiling, YU Aiwei, KANG Xuejing, and MING Anlong* \\ School of Computer Science, Beijing University of Posts and Telecommunications, Beijing 100876, China
}

\begin{abstract}
In this paper, a method to infer global depth ordering for monocular images is presented. Firstly a distance metric is defined with color, compactness, entropy and edge features to estimate the difference between pixels and seeds, which can ensure the superpixels to obtain more accurate object contours. To correctly infer local depth relationship, a weighting descriptor is designed that combines edge, T-junction and saliency features to avoid wrong local inference caused by a single feature. Based on the weighting descriptor, a global inference strategy is presented, which not only can promote the performance of global depth ordering, but also can infer the depth relationships correctly between two non-adjacent regions. The simulation results on the BSDS500 dataset, Cornell dataset and NYU 2 dataset demonstrate the effectiveness of the approach.
\end{abstract}

Keywords: superpixel segmentation, depth ordering inference, weighting descriptor.

DOI: $10.21629 / J S E E .2019 .06 .04$

\section{Introduction}

Depth ordering inference on a monocular image, also known as the 2.1D sketch, was firstly introduced by Nitzberg [1]. It decomposes an image into many nonoverlapping regions by occlusion edges and then infer their global depth relationships based on geometric features and foreground-background assignment. Depth ordering inference has many practical applications, such as robot vision [2], visual object tracking [3,4], scene understanding [5,6], object recognition $[7,8]$ and geoscience $[9,10]$. While it is a natural ability for humans to get depth relationships of different objects from a monocular image, it is still a challenging task for computer vision.

In recent years, some scholars have focused on this promising research direction. It is believed that the essential problems of depth reasoning or the 2.1D sketch are

\footnotetext{
Manuscript received December 08, 2018.

*Corresponding author.

This work was supported by the National Natural Science Foundation of China (61701036).
}

occlusion edges extraction and depth ordering inference [11 - 15]. For the occlusion edges extraction process, Amer [16] and Yu [2] obtained occlusion edges by setting an over-segmentation scale based on global probability of boundary-oriented watershed transform-ultrametric contour map (gPb-OWT-UCM) [15] and got promising results on depth ordering inference. Hoiem [13] introduced an occlusion recovery strategy, which utilized several lowlevel and high-level cues to train a conditional random field to extract occlusion edges. In addition to Hoiem, Jia [17] proposed new features based on bag-of-word [8] for occlusion boundary detection. However, their oversegmentation methods only apply color and compactness term to get objects' edges, which are not accurate enough and will lead to wrong objects' contours when extracting occlusion edges. Zhou [18] utilized a Huber Loss to extract occlusion edges and get promising results. As for deep learning methods in recent years [19-21], the occlusion edges cannot form a closed shape, which cannot satisfy our purpose.

As for the depth ordering inference, both Amer [16] and $\mathrm{Yu}$ [2] employed some geometric cues to calculate the global energy function and utilized the global optimization algorithm to obtain final depth ordering. Chen [6] introduced a training method to obtain the depth relationships of different regions based on the semantic image. Jia [17] employed a scale-invariant feature transform (SIFT) like T-junction feature and the convexity of edge feature to infer local depth relationship, through adding these features into a Markov random field. Jia also obtained global depth ordering effectively. To simplify the inference process, Ming [11] proposed a triple descriptor which combines two T-junction features and an edge feature to infer local depth order and employed the directed graph strategy to acquire final depth ordering. However, the triple descriptor will cause a wrong inference when two T-junction features are inaccurate, and the existing graph-based strategy [11,17] to infer global depth ordering is not well enough when two 
objects are separated. Different from the method of depth estimation [22-26], the depth ordering focus on objectlevel inference rather than pixel-level inference like depth estimation.

In this paper, we propose a method for depth ordering inference. It mainly consists of three aspects: a distance metric is firstly defined with color, compactness, entropy and edge features to measure the difference between pixels and seeds. In our distance metric, we set an adaptive coefficient to control the relationship between color and compactness, which can make our superpixel segmentation algorithm concentrate more on detecting edges. After the extraction of occlusion edges, a weighting descriptor that combines different weights of T-junction, edge and saliency features is introduced to overcome the limitations of Ming's triple descriptor [11] and get local depth relationship correctly. Then, we propose an inference strategy based on our weighting descriptor which not only enhance the final result of global depth ordering inference, but also correctly infer depth relationships between two separated objects. Simulation results demonstrate that our method

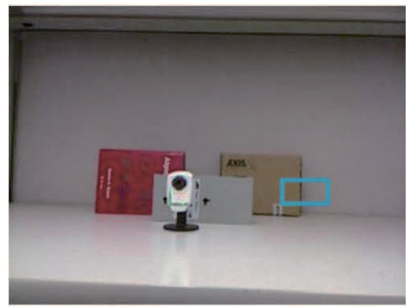

(a) Original image from Cornell cataset

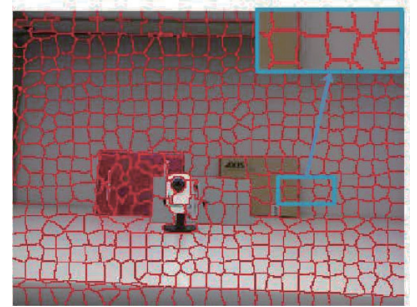

(c) Superpixels generated by our method

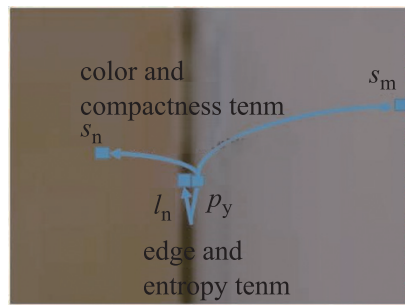

(b) Illustration of our distance metric

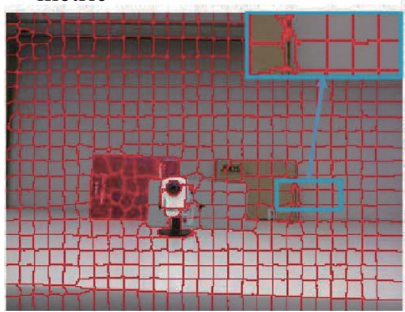

(d) Superpixels generated by SNIC [30]

perform well on the BSDS500 dataset, Cornell dataset and NYU 2 dataset.

\section{Extraction of occlusion edges}

In this section, we define a distance metric with entropy, edge, color and compactness terms to generate superpixels and then extract occlusion edges reliably.

Given a monocular image $I$, its superpixels are defined as $I=\left\{S_{1}, S_{2}, \ldots, S_{N}\right\}$, where $S_{i} \cap S_{j}=\varnothing$ and $i \neq j$. The method of simple non-iterative clustering (SNIC) [12] only considers color and compactness terms, its distance metric is

$$
D\left(s_{n}, p_{y}\right)=C\left(s_{n}, p_{y}\right)+\theta \times d\left(s_{n}, p_{y}\right)
$$

where $s_{n}$ is the seed pixel in superpixels, $\theta$ is a constant number, $C\left(s_{n}, p_{y}\right), d\left(s_{n}, p_{y}\right)$ are color and compactness terms respectively. However, in monocular images, as Fig. 1(b) and Fig. 1(d) show, the distance between $s_{n}$ and $p_{y}$ is much closer than the distance from $s_{m}$ to $p_{y}$, that is,

$$
D\left(s_{n}, p_{y}\right)<D\left(s_{m}, p_{y}\right) .
$$

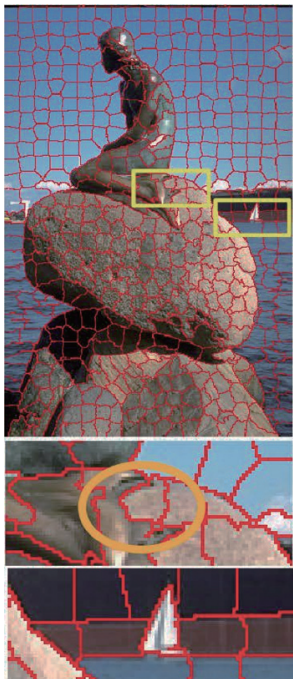

(e) Superpixels generated by our method on BSDS500 dataset

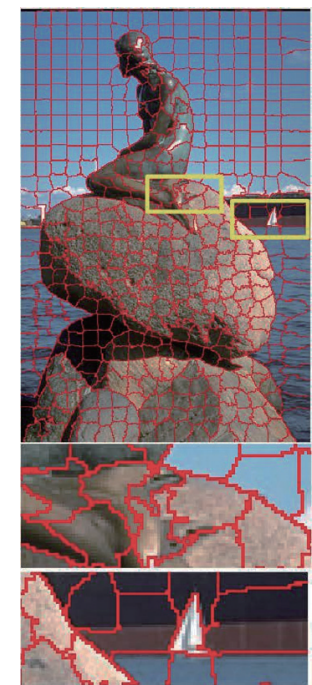

(f) Superpixels generated by SNIC [31] on BSDS500 dataset

Fig. 1 Illustration of our distance metric and superpixel segmentation maps by different methods on different datasets

This situation will cause a wrong result: pixel $p_{y}$ belongs to $s_{n}$, the edges extracted will be not accurate enough.

The entropy rate can describe the similarity of adjacent pixels efficiently and the edge term can promote the accuracy of the superpixel segmentation $[27,28]$. Therefore, we employ color and compactness as our main term to generate superpixels and add entropy and edge terms to constraint the distance between pixels and seeds, which can further improve the performance of our algorithm. In addition, we set an adaptive coefficient to control the weights between color and compactness terms, the proposed algorithm can then concentrate more on object boundaries than SNIC: the weights of compactness terms will increase when two pixels have different colors absolutely (like boundaries), and when two pixels have similar color, the weights of compactness terms will decrease. We define the distance metric as

$$
\begin{gathered}
D\left(s_{n}, l_{n}, p_{y}\right)=\alpha \times E R\left(l_{n}, p_{y}\right)-\beta \times E\left(l_{n}, p_{y}\right)- \\
\gamma \times\left(C\left(s_{n}, p_{y}\right)+\delta \times d\left(s_{n}, p_{y}\right)\right)
\end{gathered}
$$


where $l_{n}$ is the labeled pixel in $s_{n}, s_{n}$ is the seed pixel in superpixels and $p_{y}$ is the adjacent pixel with $l_{n}$. $E R\left(l_{n}, p_{y}\right)$ is the entropy term, and $E\left(l_{n}, p_{y}\right)$ is the edge term obtained by lazy random walks (LRW) [29]. $\alpha, \beta$ and $\gamma$ are three positive constant weighting coefficients whose values will be specified in Section 4. Next, we will introduce every term in our distance metric in detail.

\subsection{Color and compactness terms}

In previous work, the SNIC [12] employed a constant coefficient to control the relationship between color and compactness terms as in (1). It will obtain a wrong segmentation result when the initial seed pixel is very close to the boundary of objects. Like Fig. 1(b) shows, the pixel $p_{y}$ is much closer to the seed $s_{n}$ than to the seed $s_{m}, p_{y}$ can be easily judged as the same label with $s_{n}$. To overcome this drawback, we design an adaptive coefficient $\xi$ to make superpixels detect edges more accurately. We define

$$
\begin{gathered}
C\left(s_{n}, p_{y}\right)=\left\|L A B_{S_{n}}-L A B_{p_{y}}\right\|_{2} \\
d\left(s_{n}, p_{y}\right)=\left\|s_{n}-p_{y}\right\|_{2} \\
\delta=\theta \mathrm{e}^{-\frac{\xi}{C\left(s_{n}, p_{y}\right)+\lambda}+1}
\end{gathered}
$$

where $L A B_{p_{y}}$ is the corresponding CIELAB color of pixel $p_{y},\left\|s_{n}-p_{y}\right\|_{2}$ means the Euclidean distance of $s_{n}$ and $p_{y} \cdot \xi=\sqrt{\frac{\operatorname{size}(I)}{N}}$ is a balancing coefficient to control the scale of $\theta$, where size $(I)$ is the number of pixels in $I . \lambda=$ $10^{-4}$ is a constant number to prevent $C\left(s_{n}, p_{y}\right)+\lambda=0$. If the seed $s_{n}$ and pixel $p_{y}$ have similar color, the impact of the compactness term will be decreased. If their colors are different obviously, the coefficient $\delta$ will be larger than the constant number in SNIC, that is, $D\left(s_{n}, p_{y}\right)$ will become larger, and the drawback in SNIC can be corrected.

\subsection{Entropy term}

Because the existing superpixel segmentation methods $[12,30]$ mainly consider the information of the region and the corresponding candidate pixel, the boundary recall is not satisfactory enough (see in Section 4). We employ the static distribution of LRW to build the entropy term of each adjacent pixel in $I$ :

$$
\begin{gathered}
P(i, j)=\left\{\begin{array}{l}
\frac{w_{i j}}{w_{i}}, \quad i \neq j \\
\sum_{\alpha-\frac{j: e_{i, j} \in L}{w_{i}} w_{i j}}, i=j
\end{array}\right. \\
E R(i, j)=-\sum_{i j} u_{i} P_{i j} \log _{2} P_{i j}
\end{gathered}
$$

where $w_{i j}$ is the Gaussian weighting function as LRW, $w_{i}=\sum_{i=1}^{4} w_{i j}+w_{i i}$ is the sum of weights of pixel $p_{i}$. $u_{i}=\frac{w_{i}}{\sum w_{i}}$ is the stationary distribution in LRW. Following Liu's work [27], the entropy rate can measure the difference of adjacent pixels and describe the similarity between the labeled region and candidate pixels. The selfloops in LRW has the merits of segmenting weak boundaries and complicated texture regions well [29]. Fig. 1(e) and Fig. 1(f) are superpixel segmentation maps generated by our method, which use LRW and RW in entropy terms respectively. It is obvious that our algorithm with LRW can detect weak boundaries well.

\subsection{Edge term}

To further improve the accuracy of detecting object edges, we employ LRW to calculate the normalized transition probability map $T I$ and the edge term in our distance metric, that is,

$$
E\left(l_{n}, p_{y}\right)=\left\|\widetilde{t}_{l_{n}}-\widetilde{t}_{p_{y}}\right\|_{2}
$$

where $\widetilde{T}=1-T I$ is the probability of each pixel being an edge. In order to preserve the boundary of objects and reducing the influence of noisy pixel $\widetilde{t}_{i}$ in $\widetilde{T}$, when $\widetilde{t}_{i}<0.25$, we set $\widetilde{t}_{i}=0$. This simple term can constraint superpixels to detect edges effectively. In Fig. 1(b), $l_{n}$ is the adjacent pixel with $p_{y}$, if $l_{n}$ is an edge pixel in $\widetilde{T}, E\left(l_{n}, p_{y}\right)$ will prevent $p_{y}$ to be the same label as $l_{n}$.

Fig. 1(e) shows superpixels generated by our method and Fig. 1(d), Fig. 1(f) are superpixels generated by SNIC [30]. It is obvious that our method can detect objects contours more precisely.

After the definition of distance metric, the priority queue $Q_{u}$ [31] is employed to generate the over-segmentation map $\widetilde{I}$. Following Ming's work [11], we utilize the adaptive feature subspace learning to extract occlusion edges from $\widetilde{I}$. The occlusion edges map is defined as $\Gamma=$ $\left\{R_{1}, R_{2}, \ldots, R_{Q}\right\}$, where $R_{q}$ is the region generated by occlusion edges. In the next section, we apply occlusion edges to infer depth ordering of monocular images.

\section{Depth ordering inference}

In this section, we firstly introduce our weighting descriptor and use it to infer local depth relationships between two adjacent regions. Then, by assigning different weights to our weighing descriptor, the global inference strategy is proposed to obtain the final depth ordering.

\subsection{Local depth ordering inference based on the proposed weighting descriptor}

Ming [11] introduced a tripe descriptor to infer depth rela- 
tionships between adjacent regions. However, for the situation that two T-junctions have wrong local inference as shown in Fig. 2(a), the triple descriptor would cause wrong result: T-junction $a$ and T-junction $b$ with edge $e_{2}$ will infer that region $\mathrm{E}$ is in front of $\mathrm{C}$.

To solve this problem, we define a weighting descriptor:

$$
\Upsilon_{m}=\left(\omega a_{1}, \omega a_{2}, \psi e, \zeta \widehat{S}\right) \text { s.t. } \psi>\omega>\zeta
$$

where $m$ is the number of weighting descriptors, $\psi, \omega, \zeta$ are weighting descriptors of different features. Because the $\mathrm{T}$-junction feature may have some wrong local inference, the saliency feature can find the background region, the convexity of edge feature can be the strong feature to infer local relationship, and different values should be set to make weighting descriptors correctly. After evaluating several situations: $\psi=\omega=\zeta, \psi>\omega>\zeta$ and $\psi>\zeta>\omega$, we employ $\psi>\omega>\zeta$. Because $\psi$ should be smaller than $\omega+\zeta$ to guarantee the edge feature can be constrained by other two features, and to avoid T-junctions' error, $2 \omega$ should be smaller than $\psi+\zeta$. In our experiment, we set $\psi=0.5, \omega=0.35, \zeta=0.3$. We utilize two T-junctions $\left(a_{1}, a_{2}\right)$, an edge $e$ and saliency features $\widehat{S}$ [32] to judge the local foreground-background relationships between adjacent regions (Fig. 2(b)). When the edge and saliency features have the same inference, it can exclude wrong inference by T-junctions. In addition, if the convexity of the edge cannot infer the local relationship (straight lines), we will exclude the wrong edge feature. For example in Fig. 2(a), $e_{1}$ is a straight line that cannot correctly infer the local depth ordering, we then decrease the weights of weighting descriptor $\Upsilon_{1}$ from 1 to 0.9 .

Based on our weighting descriptor $\Upsilon_{m}$ and the graph based algorithm in [11], the directed graph Fig. 2(d) of Fig. 2(a) can be obtained. Compared with Ming's triple descriptor [11] (Fig. 2(c)), our descriptor can correctly infer region $\mathrm{C}$ is in front of $\mathrm{E}$. Because our weighting descriptor can employ local features better than the triple descriptor. To exclude the contradict inference by different weighting descriptors, different weights can be assigned to each descriptor to build the local depth relationships, if the descriptor has useless feature (like straight line), the weighting of the descriptor will be decreased.

For example, in Fig. 2(a), descriptor $\Upsilon_{1}$ has one useless feature (its corresponding edge is a straight line). We assign its weight as 0.9 . Features in $\Upsilon_{3}$ are all useful, so we assign its weight as 1 . Then, the directed depth order graph $G[11]$ is employed to exclude contradict local inference. Since we have assigned different weights to each $\Upsilon_{m}, \Upsilon_{1}$ infers that region $\mathrm{E}$ is in front of regions $\mathrm{B}$ and $\mathrm{D}, \Upsilon_{3}$ infers that region $\mathrm{B}$ is in front of region $\mathrm{E}$, and weights $\Upsilon_{1}<\Upsilon_{3}$, the directed path $\mathrm{E}$ to $\mathrm{B}$ can be removed. $\Upsilon_{4}$ and $\Upsilon_{5}$ infer that region $\mathrm{D}$ is in front of region $\mathrm{E}$, so the directed path $E$ to D in Fig. 2(d) can be removed. Our strategy of removing extra directed paths can avoid the wrong result of global depth ordering inference. The final local depth relationship is shown in Fig. 2(e).

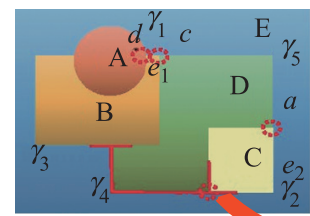

(a) Toy examples

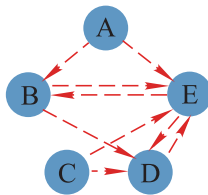

(d) Depth-order map generated by our weighting descriptor

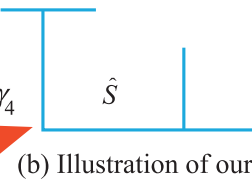

(b) Illustration of our weighting descriptor

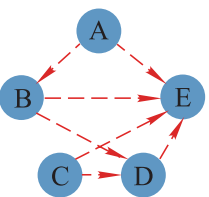

(e) Depth-order map after excluding contradict inference

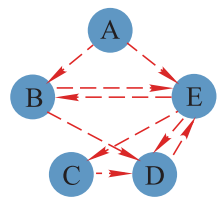

(c) Depth-order map generated by triple descriptor [11]

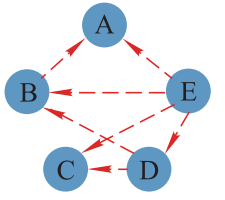

(f) Depth-order map after reversing all valid paths

Fig. 2 Illustration of the proposed weighting descriptor and the depth-order map generated by different weighting descriptors

\subsection{Global depth ordering inference}

If we use the triple descriptor in [11] to obtain the global depth ordering, region $\mathrm{C}$ and region $\mathrm{A}$ are separated, and there is no feature to infer two separated regions. For example, if node $\mathrm{A}$ is regarded as the start node, node $\mathrm{C}$ cannot be traversed by finding the longest path. If we regard the other node as the start node, the similar results will ap- pear either. In this situation, our proposed strategy can be effective. Since the background is at the bottom of the image, we firstly reverse all directed paths in $G$ (Fig. 2(f)), the ground region can then be regarded as the start node. If the node does not have the path to the other node, e.g., node $\mathrm{A}$ and node $\mathrm{C}$ do not have the path to the other node, it can be regarded as the top node in the graph. Then we 
find the longest path between the top node and the start node until all top nodes being calculated. For example, two paths in our example can be searched in Fig. 2(f), $\mathrm{E} \rightarrow \mathrm{D} \rightarrow \mathrm{B} \rightarrow \mathrm{A}$ and $\mathrm{E} \rightarrow \mathrm{D} \rightarrow \mathrm{C}$. The final result will be $1 \rightarrow 2 \rightarrow 3 \rightarrow 4$ and $1 \rightarrow 2 \rightarrow 3$, which means region $\mathrm{C}$ and region $\mathrm{B}$ are on the same layer. Finally, $\mathrm{A} \rightarrow \mathrm{B}=\mathrm{C} \rightarrow \mathrm{D} \rightarrow \mathrm{E}$ is the correct result of global depth ordering. The following is the algorithm of depth ordering inference:

Algorithm 1 Depth ordering inference

Require Input image $I$, number of seeds $N_{c}$.

Ensure The final result of depth ordering $\widehat{D}$.

Step $1 \quad I->$ Distance metric $D\left(s_{n}, l_{n}, p_{y}\right)$ priority queue $Q_{u}->$ Over-segmentation map $\widetilde{I}$;

Step $2 \widetilde{I}->$ Ming's method to extract occlusion edges [11] $->$ Occlusion edges map $\Gamma$;

Step $3 \quad \Gamma->$ Weighting descriptors

$\Upsilon_{m}=\left(\omega a_{1}, \omega a_{2}, \psi e, \zeta \widehat{S}\right)->$ The directed graph $G$;

Step $4 G->$ Inference strategy $->\widehat{D}$;

Step 5 Return $\widehat{D}$.

\section{Experimental results}

In this section we firstly validate the boundary recall (BR), under-segmentation error (UE) and running time of the superpixel segmentation method on the BSDS500 dataset [15]. As for depth ordering inference, the accuracy of results is verified on the Cornell depth-order dataset [33] and the NYU 2 dataset [34]. All datasets are performed on an Inter(R) Core(TM) i7-6700 PC $3.40 \mathrm{GHZ}$ with 8 G RAM.

\subsection{Superpixel segmentation algorithm evaluation}

To provide a fair comparison of superpixel segmentation, we employ the BSDS500 dataset and the method in [35] to evaluate our superpixel segmentation method by comparing with four other algorithms: simple linear iterative clustering (SLIC) [31], SNIC [12], linear spectral clustering (LSC) [36] and entropy rate superpixel (ERS) [37]. In addition, we also provide qualitative results to prove the effectiveness of our approach. The calculation of the entropy term utilizes the transition probability of all images, which makes the value of entropy be very small (about $1 \times 10^{-5}$ ). While for color and compactness terms, the value range is in $[0,256]$. In order to make all term values be scaled in $[0,1]$, we set the experimental parameters as $\alpha=10000$, $\beta=1, \gamma=0.04$.

\subsubsection{Quantitative results}

To evaluate the impact of different terms in our algorithm, we supply the comparison with: our method without adaptive coefficient (Ours/no-coefficient), our method with RW in entropy term (Ours/RW) and our method without edge term (Ours/no-edge) in BR and UE. Fig. 3 shows the quantitative results on boundary recall, under-segmentation error and running time by Stutz [35].

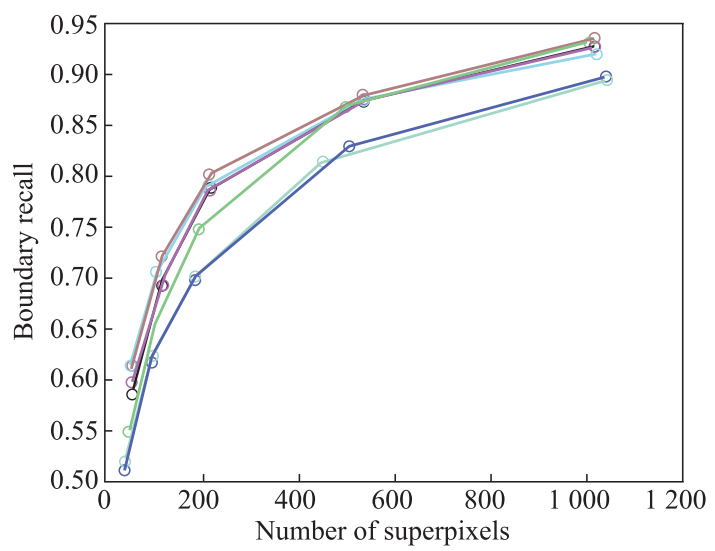

(a) Boundary recall

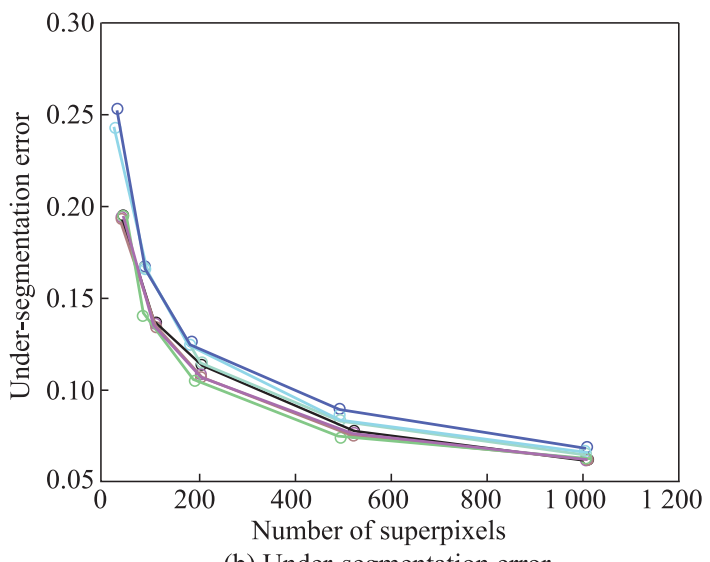

(b) Under-segmentation error

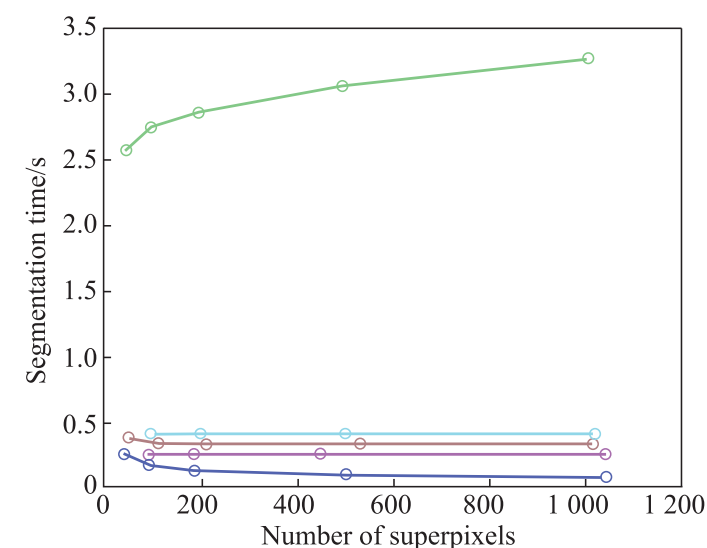

(c) Running time

$\multimap$ : ERS; - : LSC; $\multimap$ : SLIC; $\multimap$ : SNIC; $\odot$ : Ours;

$\multimap$ : Ours/no-coefficient; $\odot$ : Ours/RW; $\multimap$ : Ours/no-edge.

Fig. 3 Comparison between our algorithm and other methods

(i) Boundary recall

It is the most commonly used metric to measure the fraction of the ground truth edges overlap within a small disk 
shaped $(2 r+1) \times(2 r+1)$ neighborhood of the superpixel boundaries where $r$ is set as 1 in this section. The boundary recall comparison between our method and the other methods is indicated in Fig. 3(a). Benefiting from the high performance on boundary recall, our algorithm can obtain the edge of objects accurately. In addition, because the proposed entropy term focuses on edges detection effectively, the results of boundary recall (BR) will also be improved.

(ii) Under-segmentation error

As mentioned in [35], under-segmentation error is defined as

$$
\mathrm{UE}(G, S)=\frac{1}{N} \sum_{G_{i}} \sum_{S_{j} \cap G_{i} \neq \varnothing} \min \left\{\left|S_{j} \cap G_{i}\right|,\left|S_{j}-G_{i}\right|\right\}
$$

where $G$ is the ground truth of segmentation. Due to these edge enhancement terms, the comparisons in Fig. 3(b) demonstrate that our algorithm has competitive results with the other state-of-the-art algorithm.

(iii) Running time

In our method, we employ the priority queue to generated superpixels. Therefore, all pixels only need to calculate once, which can save much time. As the comparisons in Fig. 3(c), our method has a competitive running speed in that high performance.

\subsubsection{Qualitative results}

Though LSC [36] and ERS [37] perform very well on quantitative results, they cannot generate uniformly superpixels sizes in amounts of images, which would make the occlusion edge extraction hard to carry on. Our superpixel segmentation algorithm can provide competitive performance and regular sizes of superpixels. Fig. 4 shows the qualitative comparison superpixels generated by ERS, LSC, SLIC, SNIC and our method, and the number of pixels generated by five methods is in the same level. To make a fair comparison of the impact of different terms, we also compare with our method without adaptive coefficient (Ours/no-coefficient), edge (Ours/no-edge) and only use RW in the entropy term (Ours/RW) in Fig. 4. The results indicate that if the adaptive coefficient is not utilized, the object with background cannot be segmented correctly. Meanwhile, if not using the edge term in our method, the weights of the entropy term will become large enough and influence the shapes of superpixels (which can lead to irregular shapes).

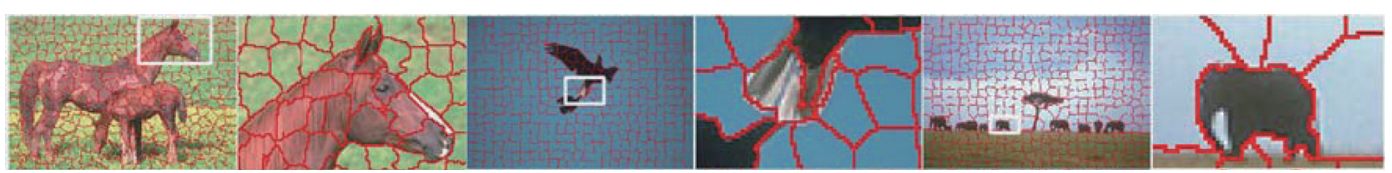

(a) Our method
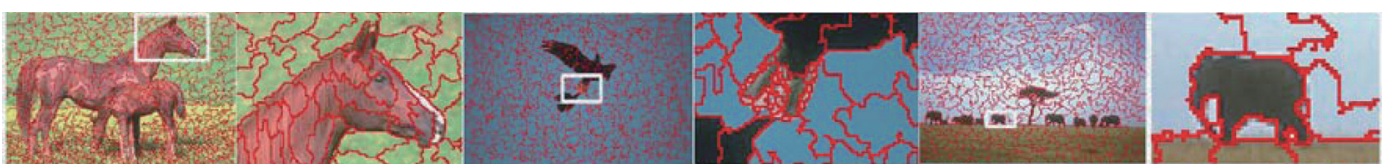

(b) ERS

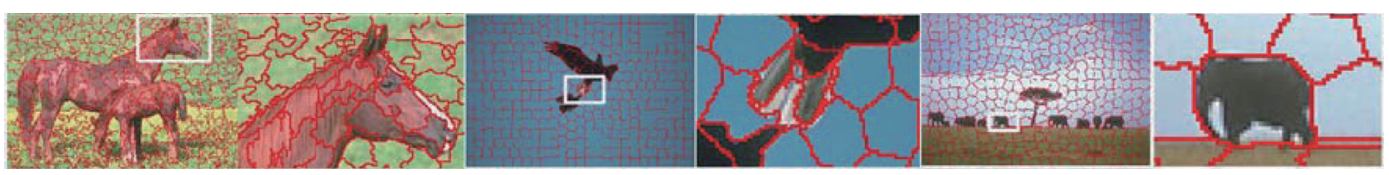

(c) LSC

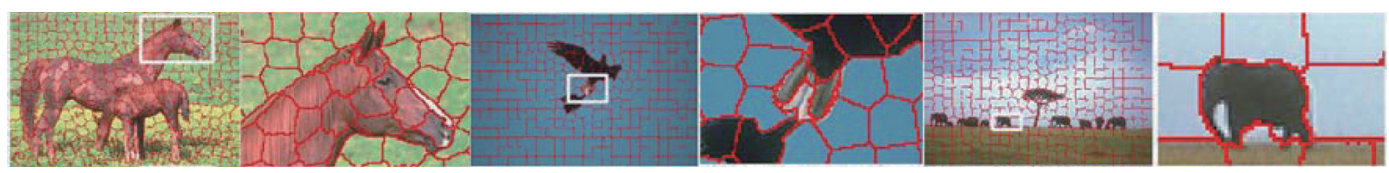

(d) SLIC

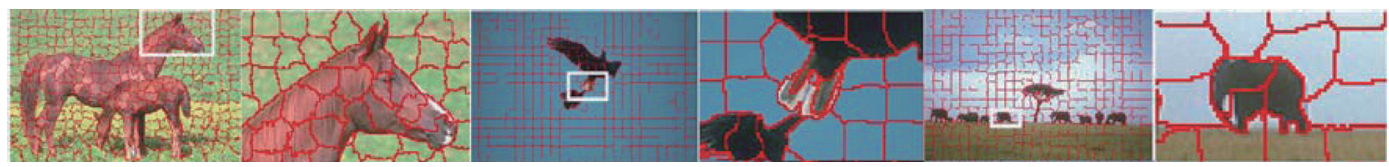

(e) SNIC 




(f) Our method without adaptive coefficient

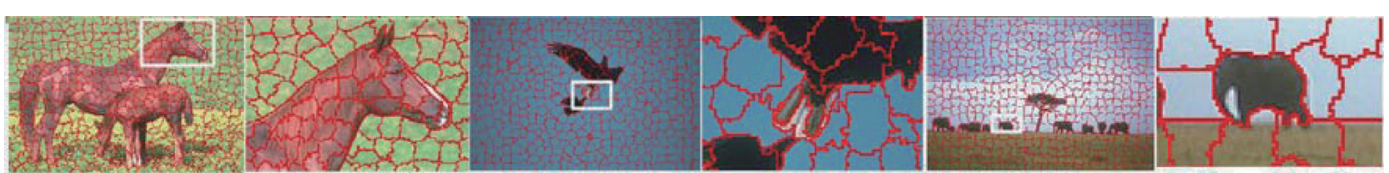

(g) Our method without edge term

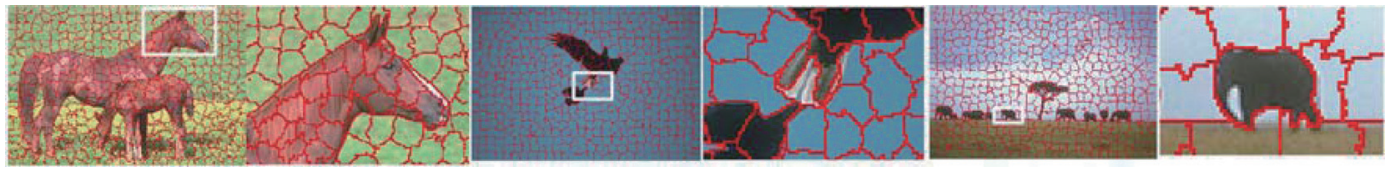

(h) RW in entropy term

Fig. 4 Qualitative comparisons

In addition, the weak boundary of objects will be hard to detect if RW is employed in the entropy term. As we can see, our method can generate regular superpixels and have high qualitative in detecting edges.

\subsection{Depth ordering evaluation}

To evaluate the results of depth ordering inference, we use Yu's method [2] as our baseline because its satisfactory result and the author also released their source code and evaluation criterion. Then, we compare it with (i) our weighting descriptor to infer local depth relationships with global inference in [2], (ii) local inference in [2] global inference by our method, (iii) Ming's method [11] to extrac- tion occlusion edges, and our method to infer depth ordering, (iv) our method to extract occlusion edges and Ming's method to infer depth ordering, (v) Ming's method. Next, we will introduce the performance of our method on different datasets.

\section{(i) Cornell depth-order dataset}

This dataset have 1087 images with many objects occluding each other in different perspectives. Based on our superpixels, Ming's method [11] is employed to extract occlusion edges. Table 1 shows the accuracy of depth ordering inference. When comparing with the baseline method [2], we have almost $13 \%$ improvement.
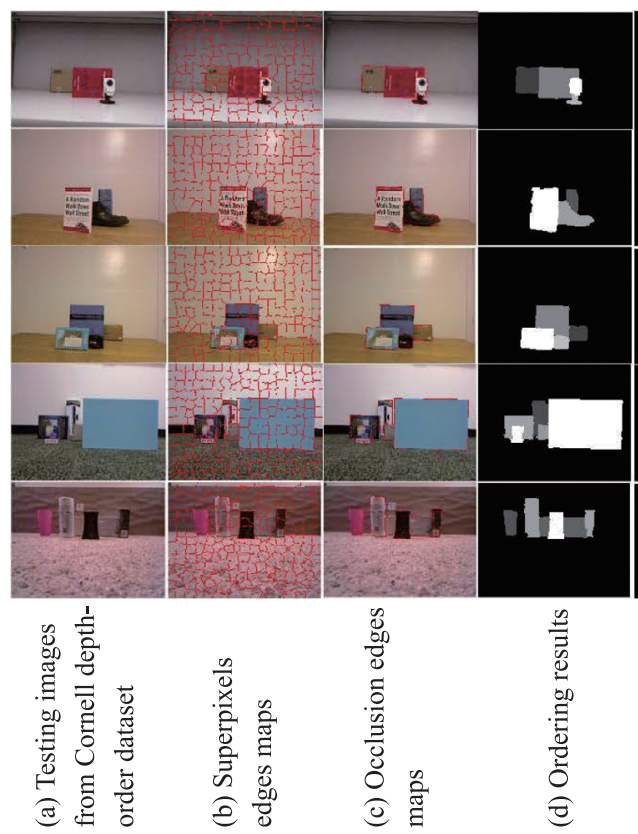
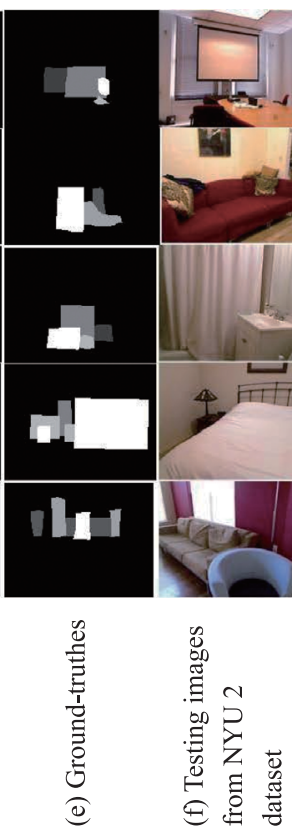
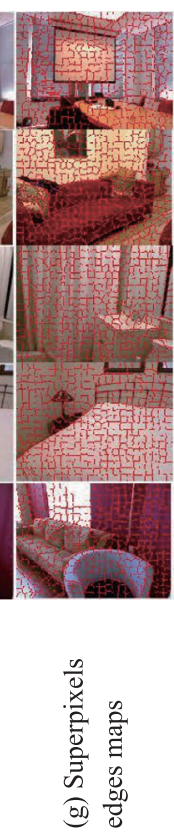
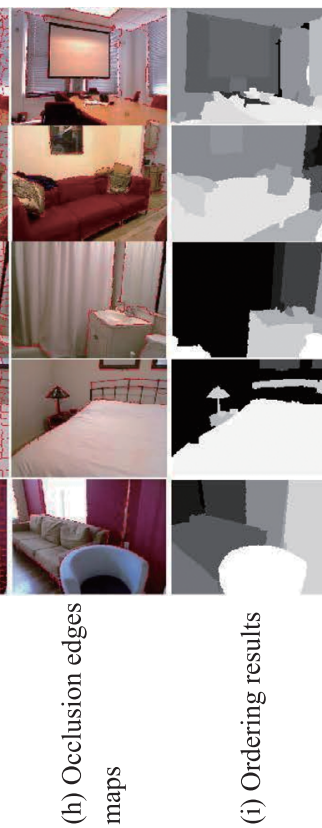

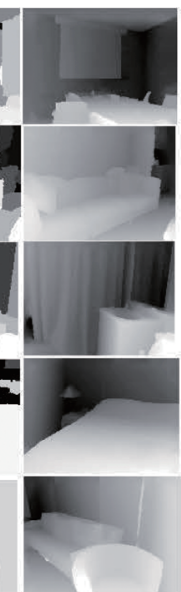

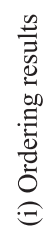

Fig. 5 Example results 
In addition, when compared with the method in [11], our weighting descriptor can improve $0.8 \%$, and our proposed method to extract occlusion edges can enhance about $0.4 \%$. Fig. 5 shows the performance of our method on the Cornell dataset. Benefiting from the accurate superpixels, the boundary of the depth ordering can describe objects' boundary more precisely. In addition, our depth inference can also infer two separated regions, which can improve our final result.

Table 1 Average depth ordering accuracy of different methods on Cornell depth-order dataset (D) and NYU 2 dataset (N)

\begin{tabular}{cccccccc}
\hline Dataset & [2] & (i) & (ii) & (iii) & (iv) & (v) & Ours \\
\hline Cornell & 79.1 & 84.6 & 84.3 & 91.8 & 91.4 & 91.0 & $\mathbf{9 2 . 1}$ \\
NYU 2 & 60.3 & 70.6 & 69.5 & 78.9 & 78.5 & 78.0 & $\mathbf{7 9 . 6}$ \\
\hline
\end{tabular}

\section{(ii) NYU dataset}

This dataset contains 1449 RGBD images. The scenes in this dataset are more complex and challenging. Table 1 demonstrates the results on this challenging dataset. Comparing with the baseline method, we achieve more than $19 \%$ improvement in accuracy. And we can also have $1.6 \%$ improvement compared with Ming's method [11]. It is mainly because our proposed weighting descriptor can describe local depth relationship better and our superpixel segmentation method can detect objects' contour more accurately. Fig. 5 shows the performance of our method on the NYU2 dataset.

\section{Conclusions}

In this work, an approach to infer depth ordering from a monocular image is proposed. Our main contributions are: we firstly propose a distance metric for the superpixel segmentation algorithm which combines edge, entropy, compactness and color terms to segment objects' contour more accurately. Secondly, a weighting descriptor is introduced that contains two T-junction features, an edge feature and saliency features to infer local depth relationships between adjacent regions robustly. Finally, an inference strategy is proposed based on our weighting descriptor, which can enhance the performance of final depth ordering inference and get the final depth relationship between two non-adjacent regions. Our approach has been applied on two challenging datasets and the experimental results demonstrate the effectiveness of our method.

\section{References}

[1] MARK N, MUMFORD D. The 2.1-d sketch. Proc. of the IEEE International Conference on Computer Vision, 1990: $138-$ 144.

[2] YU C C, LIU Y J, WU M T, et al. A global energy optimization framework for 2.1D sketch extraction from monocular images. Graphical Models, 2014, 76(5): 507-521.
[3] HONG Z, MEI X, TAO D. Dual-force metric learning for robust distracter-resistant tracker. Proc. of the European Conference on Computer Vision, 2012: 513-527.

[4] ZHOU Y, BAI X, LIU W Y, et al. Fusion with diffusion for robust visual tracking. Advances in Neural Information Processing Systems, 2012, 4: 2978-2986.

[5] KARSCH K, LIU C, KANG S B. Depth extraction from video using non-parametric sampling. Proc. of the European Conference on Computer Vision, 2012: 775-788.

[6] CHEN X, LI Q, ZHAO D, et al. Occlusion cues for image scene layering. Computer Vision and Image Understanding, 2013, 117(1): 42-55.

[7] GOULD S, FULTON R, KOLLER D. Decomposing a scene into geometric and semantically consistent regions. Proc. of the International Conference on Computer Vision, 2010: 1-8.

[8] LI F F, PERONA P. A bayesian hierarchical model for learning natural scene categories. Proc. of the IEEE Computer Society Conference on Computer Vision and Pattern Recognition, 2005: $524-531$.

[9] MA J, ZHOU H, ZHAO J, et al. Robust feature matching for remote sensing image registration via locally linear transforming. IEEE Trans. on Geoscience and Remote Sensing, 2015, 53(12): $6469-6481$.

[10] MA J, QIU W, ZHAO J, et al. Robust L2E estimation of transformation for non-rigid registration. IEEE Trans. on Signal Processing, 2015, 63(5): 1115-1129.

[11] MING A, WU T, MA J, et al. Monocular depth-ordering reasoning with occlusion edge detection and couple layers inference. IEEE Intelligent Systems, 2016, 31(2): 54-65.

[12] ACHANTA R, SUSSTRUNK S. Superpixels and polygons using simple non-iterative clustering. Proc. of the IEEE Conference on Computer Vision and Pattern Recognition, 2017: $4895-4904$.

[13] HOIEM D, EFROS A A, HEBERT M. Recovering occlusion boundaries from an image. International Journal of Computer Vision, 2011, 91(3): 328-346.

[14] FOWLKES C C, MARTIN D R, MALIK J. Local figureground cues are valid for natural images. Journal of Vision, 2007, 7(8): 1-9.

[15] ARBELAEZ P, MAIRE M, FOWLKES C, et al. Contour detection and hierarchical image segmentation. IEEE Trans. on Pattern Analysis and Machine Intelligence, 2011, 33(5): 898916.

[16] AMER M R, YOUSEFI S, RAICH R, et al. Monocular extraction of $2.1 \mathrm{~d}$ sketch using constrained convex optimization. International Journal of Computer Vision, 2015, 112(1): $23-$ 42.

[17] JIA Z. A learning-based framework for depth ordering. Proc. of the IEEE Conference on Computer Vision and Pattern Recognition, 2012: 294-301.

[18] ZHOU Y, MA J, MING A, et al. Learning training samples for occlusion edge detection and its application in depth ordering inference. Proc. of the 24th International Conference on Pattern Recognition, 2018, DOI: 10.1109/ICPR.2018.8545622.

[19] YU Z, FENG C, LIU M Y, et al. Casenet: deep categoryaware semantic edge detection. Proc. of the IEEE Conference on Computer Vision and Pattern Recognition, 2017: 1-10.

[20] PENG W, YUILLE A. Doc: deep occlusion estimation from a single image. Proc. of the European Conference on Computer Vision, 2016: 546-561.

[21] WANG G X, LIANG X H, LI F W B. Doobnet: deep object occlusion boundary detection from an image. Computer Vision and Pattern Recognition, 2018, arXiv:1806.03772. 
[22] HE L, WANG G, HU Z. Learning depth from single images with deep neural network embedding focal length. IEEE Trans. on Image Processing, 2018, 27(9): 4676-4689.

[23] FU H, GONG M, WANG C, et al. Deep ordinal regression network for monocular depth estimation. Proc. of the IEEE Conference on Computer Vision and Pattern Recognition, 2018: $2002-2011$.

[24] XU D, WANG W, TANG H, et al. Structured attention guided convolutional neural fields for monocular depth estimation. Proc. of the IEEE Conference on Computer Vision and Pattern Recognition, 2018: 3917 -3925.

[25] XU D, OUYANG W, WANG X, et al. Pad-net: multi-tasks guided prediction-and-distillation network for simultaneous depth estimation and scene parsing. Proc. of the IEEE Conference on Computer Vision and Pattern Recognition, 2018, DOI: 10.1109/CVPR.2018.00077.

[26] XU D, RICCI E, OUYANG W, et al. Multi-scale continuous CRFs as sequential deep networks for monocular depth estimation. Proc. of the IEEE Conference on Computer Vision and Pattern Recognition, 2017: 5354-5362.

[27] LIU M Y, TUZEL O, RAMALINGAM S, et al. Entropy rate superpixel segmentation. Proc. of the Computer Vision and Pattern Recognition, 2011: 2097 - 2104.

[28] ZHANG Y, LI X, GAO X, et al. A simple algorithm of superpixel segmentation with boundary constrain. IEEE Trans. on Circuits \& Systems for Video Technology, 2017, 27(7): 1502 1514.

[29] SHEN J, DU Y, WANG W, et al. Lazy random walks for superpixel segmentation. IEEE Trans. on Image Processing, 2014, 23(4): $1451-1462$.

[30] ACHANTA R, SUSSTRUNK S. Superpixels and polygons using simple non-iterative clustering. Proc. of the IEEE Conference on Computer Vision and Pattern Recognition, 2017: $4895-4904$.

[31] ACHANTA R, SHAJI A, SMITH K, et al. SLIC superpixels compared to state-of-the-art superpixel methods. IEEE Trans. on Pattern Analysis and Machine Intelligence, 2012, 34(11): $2274-2282$.

[32] CHENG M M, WARRELL J, LIN W Y, et al. Efficient salient region detection with soft image abstraction. Proc. of the IEEE International Conference on Computer Vision, 2014: 15291536.

[33] JIA Z, GALlagheR A, CHANG Y J, et al. A learning-based framework for depth ordering. Proc. of the Computer Vision and Pattern Recognition, 2012, DOI:10.1109/CVPR.2012.6247688.

[34] SILBERMAN N, HOIEM D, KOHLI P, et al. Indoor segmentation and support inference from RGBD images. Proc. of the European Conference on Computer Vision, 2012: 1-14.

[35] STUTZ D, HERMANS A, LEIBE B. Superpixels: an evalu- ation of the state-of-the-art. Computer Vision and Image Understanding, 2017: S1077314217300589.

[36] LI Z, CHEN J. Superpixel segmentation using linear spectral clustering. Proc. of the Computer Vision and Pattern Recognition, 2015: 1356-1363.

[37] LIU M Y, TUZEL O, RAMALINGAM S, et al. Entropy rate superpixel segmentation. Proc. of the Computer Vision and Pattern Recognition, 2011: 2097 -2104.

\section{Biographies}



SONG Guiling was born in 1978. He is studying for his Ph.D. degree at the School of Computer Science, Beijing University of Posts and Telecommunications, Beijing, China. His research interests are image processing and human computer interaction. E-mail: expsong@qq.com

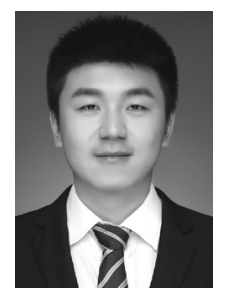

YU Aiwei was born in 1993. He is studying for his master's degree at the School of Computer Science, Beijing University of Posts and Telecommunications, Beijing, China. His research interests are image processing and computer vision.

E-mail: 2603803139@qq.com

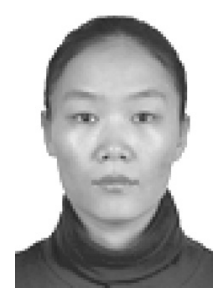

KANG Xuejing was born in 1984. She received her B.S. and M.S. degrees from Tianjin University of Technology in 2008 and 2012, respectively, and Ph.D. degree in Beijing Institute of Technology. She is currently a lecturer with the School of Computer Science, Beijing University of Posts and Telecommunications, Beijing, China. Her current research interests include fractional Fourier transform, image processing and computer vision.

E-mail: kangxuejing@bupt.edu.cn

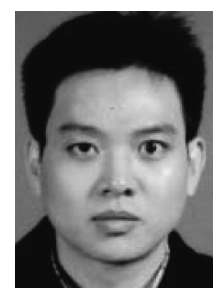

MING Anlong was born in 1979. He received his Ph.D. degree in computer science from the School of Computer Science at Beijing University of Posts and Telecommunications, China, in 2008. His research interests include multimedia systems, computer vision and image processing. E-mail: mal@bupt.edu.cn 